\title{
Psychosis and its Association with Autoimmune Disorders: A Review
}

\author{
Sumera Zaib*1 ${ }^{* 1}$ Areeba ${ }^{1}$, Nehal Rana ${ }^{1}$, Zainab Zaib ${ }^{2}$, Mohammad Afzal ${ }^{1}$ and Imtiaz Khan*3 \\ ${ }^{1}$ Department of Biochemistry, Faculty of Life Sciences, University of Central Punjab, Pakistan \\ ${ }^{2}$ Frontier Medical and Dental College, Abbottabad, Pakistan \\ ${ }^{3}$ Manchester Institute of Biotechnology, The University of Manchester, United Kingdom \\ *Corresponding author: Sumera Zaib, Department of Biochemistry, Faculty of Life Sciences, University of Central Punjab, Pakistan \\ and Imtiaz Khan, Manchester Institute of Biotechnology, The University of Manchester, United Kingdom
}

\section{ARTICLE INFO}

Received: 㓞 March 22, 2021

Published: March 30, 2021

Citation: Sumera Zaib, Areeba, Nehal Rana, Zainab Zaib, Mohammad Afzal, Imtiaz Khan. Psychosis and its Association with Autoimmune Disorders: A Review. Biomed J Sci \& Tech Res 34(5)-2021. BJSTR. MS.ID.005619.

Keywords: Bipolar Disorder; Cardiovascular Disease; Inflammatory Disorders; Schizophrenia

\section{ABSTRACT}

Psychosis is a psychological disorder that causes an imbalance in social functioning, perception, and thought. Several genetic and environmental factors are in accordance with the progression of psychosis. Schizophrenia, severe depression, and bipolar disorder causes severe mental illness psychosis. Schizophrenia is a multisystem disorder that causes several immune dysfunctions such as abnormal levels of circulating cytokines. Bipolar disorder is associated with immune dysfunctions and increases the rate of inflammatory medical comorbidities, including cardiovascular disease, chronic infections, autoimmune disorders, and metabolic disorders. In psychosis, a single relapse occurs that causes the disability of facial expressions. Emotion processing deficit persists in the acute phase of schizophrenia and the symptomatic recovery. Furthermore, psychosis acts like an autoimmune disease that leads to CNS autoimmune and inflammatory diseases, like grave's diseases, multiple sclerosis, diabetes, systemic lupus erythematosus, autoimmune vasculatures, and sarcoidosis. This review deals with the genetic association in psychosis and the relation between psychosis and autoimmune disorders.

Abbreviations: MHC: Major Histocompatibility Complex; CSF: Cerebrospinal Fluids; TNF: Tumor Necrosis Factor; IL: Interleukin, TGF: Transforming Growth Factor; BP: Bipolar Disorder; BCIS: Beck Cognitive Insight; NNAI: Non-Neurological Autoimmune; SLE: Systemic Lupus Erythematosus; GAD: Glutamic Acid Decarboxylase; GABA: GammaAminobutyric Acid

\section{Introduction}

Psychosis is a common disorder that leads to an imbalance in social functioning, perception, and thought. In the worst cases, the disease becomes life-threatening [1]. Schizophrenia is a psychotic illness commonly present in $1 \%$ of the population. The disease begins in early adulthood and persists throughout life. The common features are relapse and progression that become serious over time $[2,3]$. For the treatment, early interference with the medical and psychosocial trends is efficacious but better comprehension of the disease lowers the psychosis onset in schizophrenia [4]. Factors that cause psychosis in schizophrenia and bipolar disorder include:
1) Genetic factor,

2) Hormone change,

3) Change in brain chemicals.

Over the centuries, the relationship between autoimmune disorder and psychosis has been explored. In autoimmune diseases, the tissue damage is mediated by the $\mathrm{T}$ cells and antibodies that induce various symptoms. These symptoms affect the different body parts [5]. Recent studies have shown that the excess of cooccurrence causes two disorders to develop, such as schizophrenia 
and autoimmune diseases [6,7]. Both of the diseases are heritable and have a close association with each other [8,9]. Both diseases are immune-related to major histocompatibility complex (MHC) [9] The close association indicates that the schizophrenia involves the immune system and the genetic signals are present on chromosome $6 p$, where the MHC gene is also located [10]. Moreover, immune alteration in psychosis patients has also been observed. It indicates the elevated level of inflammatory markers in both cerebrospinal fluids (CSF) and blood and active microglia in the brain. These inflammations can cause autoimmunity and are considered as a risk factor for the psychosis $[11,12]$.

\section{Psychosis as an Autoimmune Disease}

Autoimmunity has emerged as a potential disease mechanism [13]. It focuses on adaptive immunity against psychosis. The criteria for the autoimmune disease includes 1) the evidence of diseases for particular adaptive immune response in the specific tissues, organs, or blood; 2) the passive transfer of the autoreactive cells and antibodies replicates in human by in-utero maternofoetal transfer or transplant procedures and 3) family history of autoimmune disease [14].

\section{The Relationship between Psychosis and Autoimmune Disorder}

In psychotic illness, various courses occur. These courses range from the single episode of illness to relapse and cause disability. The degree of overlap varies from time and causes central nervous system infectious disorder like multiple sclerosis [15]. Psychosis is associated with neuropsychiatric or systemic symptoms. The CNS autoimmune and inflammatory disorder leads to multiple sclerosis, systemic lupus erythematosus, autoimmune vasculatures, and sarcoidosis [16-19]. In these disorders, the mechanism of the underlying psychotic symptom remains unknown. However, systemic lupus erythematosus is an exception in which the relation between psychosis and autoantibodies has been described. But these are not proven scientific theories of lupus psychosis [20-23].

\section{Genetic Associations in Schizophrenia}

Schizophrenia is a genetic disorder having a heritability estimate of $80 \%$ [24]. At an epidemic level, increased chance of autoimmune disorder in psychoses and other psychotic disorders is found. Moreover, people suffering from the autoimmune disorder are at high risk of getting schizophrenia $[13,25]$. The diagnosis of psychotic disorder is similar to an autoimmune disorder [5]. Rheumatoid arthritis is considered the only autoimmune disease that has a negative association by schizophrenia. Individuals suffering from schizophrenia are at risk greater than $29 \%$ to get rheumatoid arthritis [26,27], however, the underlying reasons are not fully understood. A study showed a small robust negative nucleotide polymorphism relation between these disorders [28]. Psychosis shares an environmental risk factor with several other autoimmune diseases like a season of birth latitude effect associated to the newly born vitamin D status [29]

\section{Abnormal Level of Serum Cytokines in Schizophrenia}

Schizophrenia is a multisystem disorder that causes several immune dysfunctions such as abnormal levels of circulating cytokines, mediator of the immune system [30]. The cytokines are connected with behavioral activities. Any change in cytokines causes the potential implications leading to schizophrenia [31]. Serum cytokines influence psychosis in schizophrenia patients [32]. The abnormal level of cytokines is the common manifestation of schizophrenia [33]. The level of cytokines in different SHZ patients have been investigated and the results indicate an increased levels in cytokines such as tumor necrosis factor (TNF)- $\alpha$, IL-1 $\beta$, IL-12, interleukin (IL)-6, and transforming growth factor (TGF)- $\beta$ [34]. Furthermore, it was observed that symptom severity, aggression, cognitive abilities were linked with an increased level of cytokines [35]. Schizophrenia patients are more prone to sudden cardiac death [36].

\section{Schizophrenia and Bipolar Disorder with Psychosis}

In schizophrenia, facial displays of emotions are a well-known impairment [37]. Emotion processing deficit persists in the acute phase of schizophrenia and the symptomatic recovery [38]. The confusion in facial expressions is common in bipolar disorder (BP) [39]. Other neurobehavioral deficits occur in schizophrenia patients with a history of psychosis. The facial deficit becomes prominent in schizophrenia along with other disorders such as bipolar disorder [40]. Various investigations have demonstrated a greater facial deficit in SHZ compared to bipolar patients [41].

\section{Acute Psychosis in Schizophrenia and loss of Sight}

The most common effect of schizophrenia is the loss of sight. Insight psychosis causes the multidimensional concept that takes place on a continuum $[42,43]$. Clinical insight is a common diagnosis and therapy of schizophrenia. However, the concept has few drawbacks. Insight psychosis is linked with dissociation from misinterpretations and reevaluating distorted beliefs [44]. The beck cognitive insight (BCIS) was evolved to investigate the patient's condition [45]. Cognitive insight can be linked to the present symptoms and stage of disease, however, relation between clinical symptoms and cognitive insight is still unknown [46]. A recent study have demonstrated a mild relation between the negative, positive, and self-certainty scale of BCIS patients [47]. Several investigations on understanding the relationship between the cognitive insight in schizophrenia's patients with or without psychotic symptoms have been made. Moreover, the relation between acute symptoms and alteration in cognitive insight was also studied. Results showed that patients having current psychotic symptoms show careless in their perception and were damaged in self-reflectiveness which was decreased in the treatment. No effect was observed on the over-confidence after the symptomatic improvement. The overconfidence with misinterpretations is a more continues feature 
which expands beyond psychoactive states in a schizophrenia patient. The patient having no psychotic symptoms showed more self-reflectiveness than a patient having psychotic symptoms [48]. An individual with schizophrenia has overconfidence in their judgment than depressed patients, and delusions patients show high self-certainty $[49,50]$. Schizophrenia patients decide without any information and are overconfident in their decisions. Overconfidence can be influenced by personality. With better self-esteem and copying styles, the poor insight can be improved. Overconfidence is the common manifestation in delusion-prone schizophrenia individuals rather than acute psychosis [48,51-53].

\section{Psychosis and its Association with Non-Neurological Autoimmune Disorders}

The relation between psychosis and non-neurological autoimmune (NNAI) diseases has remained under investigation over the past few decades [54]. In 1950s, rheumatoid arthritis was less prevalent in general population $[55,56]$, however, a nonneurological disorder such as systemic lupus erythematosus (SLE), celiac disease, and autoimmune thyroid diseases were more widespread in psychosis patients [57]. The existence of autoimmune disorder poses a high risk of schizophrenia by $\sim 1.29$ folds. On the contrary, autoimmune disorder was observed to grow by 1.53 folds as a result of schizophrenia [58]. Schizophrenia and autoimmune disorder cause the enhanced activity of complement system [59]. Multiple factors are involved in the association between the NNAI disorder and psychosis. These factors include infection, predisposing infections, shared genetic exposure, and the brain reactive antibodies $[25,60]$. In psychosis, the level of different inflammatory markers such as C-reactive proteins and cytokines increases. Furthermore, it also causes the increase of pro-inflammatory cells, such as $\mathrm{T}$ helper 17 cells [13,61]. A study showed a positive association between schizophrenia and autoimmune disorders. Furthermore, the overall relationship between the NNAI disorders and psychosis has been monitored by using meta-analytic techniques. The result indicated that NNAI disorders causes the psychiatric manifestation of the neurological auto-immune diseases. Moreover, the primary investigation showed the effect size acquired from the different studies. The overall consequence size was small and heterogeneity was determined indicating the positive relation between the designs of study and outcomes of psychosis. The study was performed separately for the NNAI resulted a positive association for different disorders like Graves' disease pemphigoid, psoriasis, celiac disease, and anemia. The negative association between the rheumatoid arthritis and ankylosing spondylitis was detected [62,63].

\section{Psychosis and its Association with Autoimmune Disorders}

The prevalence of autoimmune disease in the general population is about $4 \%$ while the prevalence of schizophrenia is around $1 \%$
[64]. In a hospital survey, it was seen that $3.4 \%$ population with autoimmune disorders had contact with schizophrenia during their follow-up period [65]. Furthermore, an increase of $70 \%$ in bipolar disorder was detected during the 5 years after exposure to autoimmune disorder and prognosis [25]. Schizophrenia has a positive association with different autoimmune diseases such as celiac disease, pernicious anemia, grave disease, psoriasis, hypersensitivity vasculitis, and the negative relationship with the rheumatoid arthritis [27].

\section{Psychosis and its Associations with Celiac Disease}

Investigations at clinical level have found the abundance of celiac disease in $2.1-2.6 \%$ of patients having schizophrenia as compared to $0.3 \%$ to $1 \%$ population [66]. The intake of gluten causes production of transglutaminase and antigliadin antibodies by the immune system [67]. In the presence and absence of autoimmune diseases, a wide variety of neurological issues is linked with gliadin $[68,69]$. A recent finding indicated the presence of self-antigen tissue transglutaminase that is characteristic of celiac disease. In 1950s and 1960s, a close association between celiac diseases and schizophrenia was observed [70,71]. The epidemiologic studies indicated the increased occurrence of celiac disease in patients of schizophrenia. A study represented the amplified prevalence of schizophrenia in population who consumed more grains. Thus, the whole study concluded the valuable outcome of a gluten-free diet in the symptoms of schizophrenia having celiac disease and gluten sensitivity [72-74].

\section{Psychosis and its Association with Rheumatoid Arthritis}

Several studies showed the negative association of rheumatoid arthritis with psychosis. The disease onset for rheumatoid arthritis is less as compared to schizophrenia and bipolar disorder [75]. The incidence of rheumatoid arthritis lowers/raises the risk of bipolar disorder and schizophrenia [76,77]. Several autoimmune diseases are associated with rheumatoid arthritis that occurs due to genetic influences. Several markers in the major histocompatibility complex region are involved with psychiatric disorders [5]. A study indicated the role of interleukin 1 antagonist that causes the elevation in schizophrenia but is protective against rheumatoid arthritis [77]. In the negative association, anti-inflammatory and analgesic effect is involved. Furthermore, bipolar disorder has no association with rheumatoid arthritis [78,79]. However, the increased incidence was seen within 5 years of prognosis of rheumatoid arthritis [80].

\section{Psychosis and its Association with Autoimmune Disorders of Thyroid Glands}

Auto-immune thyrotoxicosis (Graves' disease) and autoimmune thyroiditis have an association and increases the risk of schizophrenia in the general population [27]. Furthermore, autoimmune thyroid disease has psychiatric symptoms associated with anti-thyroid antibodies [81]. In a study, positive family history 
was detected between thyroiditis and autoimmune thyrotoxicosis with schizophrenia $[27,82]$.

\section{Psychosis and its Association with Diabetes}

In diabetes type-I, the autoantibody against glutamic acid decarboxylase (GAD) causes the affinity towards GAD that is expressed in the brain [83]. GAD is complicated in development of gamma-aminobutyric acid (GABA) (a central neurotransmitter) $[79,84]$. GAD antibodies are linked with neurological disorders, include stiff-person syndrome. According to the survey, the occurrence of diabetes type-I is enhanced in patients of schizophrenia prior to diagnosis and later. Three different studies recommended the positive family history among diabetes type-I and schizophrenia [25].

\section{Psychosis and its Association with Systemic Lupus Erythematosus}

Almost $40 \%$ of neuropsychiatric systemic lupus erythematosus (SLE) indications developed before the SLE onset or at diagnosis time. Moreover, about $60 \%$ of manifestations occur in the 1 st year of diagnosis. Current evidence showed that $14 \%$ and $75 \%$ of SLE experience symptoms of neuropsychiatry include psychosis. Brain reactive antibodies have a defined role in developing neuropsychiatric symptoms. These antibodies have an affinity towards the anti-N-methyl-D-aspartate glutamate receptor within brain and are known to be the current pathophysiological theories of psychosis. In another study, it was confirmed that SLE patients have also increased association with non-effective psychosis $[13,85]$.

\section{Psychosis and its Association with Multiple Sclerosis}

Multiple sclerosis causes the large-scale infiltration of the immune system cells in the brain parenchyma and activate the resident inflammatory nerve damage. It is linked with the high risk of schizophrenia, bipolar disorder, and non-effective psychosis. Furthermore, the family history between the multiple sclerosis and schizophrenia with non-effective psychosis was detected [86-89].

\section{Conclusion}

Psychosis is a serious mental illness that causes the hallucination and delusions. Furthermore, it also leads to personality change. It occurs in three phases such as prodromal phase, acute phase, and recovery phase. There is a positive relationship between several auto-immune disorders and antibodies with schizophrenia. The relationship of numerous auto-immune disorders observed in the accumulation with psychosis, together with positive relationship of auto-immune thyrotoxicosis, pernicious anemia, and psoriasis, and the negative relationship with the rheumatoid arthritis. Moreover, a close association of family history and genetic factors contributes to the development of psychosis. We have highlighted genetic association of several autoimmune diseases has been explained with schizophrenia in more details in reference to abnormal level of serum cytokines. It is therefore concluded that for the treatment of psychotic patients, medications and therapies are possible that offer their overall recovery.

\section{Conflict of Interest}

The authors declare no conflict of interest, financial or otherwise.

\section{Acknowledgement}

We acknowledge the support of Department of Biochemistry, University of Central Punjab, Lahore, Pakistan.

\section{References}

1. Al Diwani AA, Pollak TA, Irani SR, Lennox BR (2017) Psychosis: an autoimmune disease? Immunology 152(3): 388-401.

2. Andrade LH, Wang YP (2012) Prevalence of psychotic symptoms in the general population varies across 52 countries. Evidence-Based Mental Health 15(4): 105.

3. Owen MJ, Sawa A, Mortensen PB (2016) Schizophrenia. Lancet 388(10039): 86-97.

4. Grande I, Berk M, Birmaher B, Vieta E (2016) Bipolar disorder. Lancet 387(10027): 1561-1572.

5. Benros ME, Mortensen PB, Eaton WW (2012) Autoimmune diseases and infections as risk factors for schizophrenia. Annals of the New York Academy of Sciences 1262: 56-66.

6. Davidson A, Diamond B (2001) Autoimmune diseases. The New England Journal of Medicine 345(5): 340-350.

7. Sullivan PF, Kendler KS, Neale MC (2003) Schizophrenia as a complex trait: Evidence from a meta-analysis of twin studies. Archives of General Psychiatry 60(12): 1187-1192.

8. Cho JH, Gregersen PK (2011) Genomics and the multifactorial nature of human autoimmune disease. The New England Journal of Medicine 365: 1612-1623.

9. Jones AL, Mowry BJ, Pender MP, Greer JM (2005) Immune dysregulation and self-reactivity in schizophrenia: Do some cases of schizophrenia have an autoimmune basis? Immunology and Cell Biology 83(1): 9-17.

10. (2011) Schizophrenia Psychiatric Genome-Wide Association Study (GWAS) Consortium. Genome-wide association study identifies five new schizophrenia loci. Nature Genetics 43(10): 969-976.

11. Nikkila HV, Muller K, Ahokas A, Rimon R, Andersson LC (2001) Increased frequency of activated lymphocytes in the cerebrospinal fluid of patients with acute schizophrenia. Schizophrenia Research 49(1-2): 99-105.

12. Miller BJ, Gassama B, Sebastian D, Buckley P, Mellor A (2013) Meta-analysis of lymphocytes in schizophrenia: Clinical status and antipsychotic effects. Biological Psychiatry 73(10): 993-999.

13. Benros ME, Eaton WW, Mortensen PB (2014) The epidemiologic evidence linking autoimmune diseases and psychosis. Biological Psychiatry 75(4): 300-306.

14. Pillinger T, Beck K, Gobjila C, Donocik JG, Jauhar S, et al. (2017) Impaired glucose homeostasis in first-episode schizophrenia: a systematic review and meta-analysis. JAMA Psychiatry 74(3): 261-269.

15. Camara Lemarroy CR, Ibarra Yruegas BE, Rodriguez Gutierrez R, Berrios Morales I, Ionete C, et al. (2017) The varieties of psychosis in multiple sclerosis: a systematic review of cases. Multiple Sclerosis and Related Disorders 12: 9-14.

16. Gilberthorpe TG, O' Connell KE, Carolan A, Silber E, Brex PA, et al. (2017) The spectrum of psychosis in multiple sclerosis: a clinical case series. Neuropsychiatric Disease and Treatment 13: 303-318. 
17. Duwe BV, Turetsky BI (2002) Misdiagnosis of schizophrenia in a patient with psychotic symptoms. Neuropsychiatry Neuropsychology and Behavioural Neurology 15(4): 252-260.

18. Zielasek J, Bender G, Schlesinger S, Friedl P, Kenn W, et al. (2002) A woman who gained weight and became schizophrenic. Lancet 360(9343): 1392

19. Talarico R, Palagini L, d'Ascanio A, Elefante E, Ferrari C, et al. (2015) Epidemiology and management of neuropsychiatric disorders in Behçet's syndrome. CNS Drugs 29(3): 189-196.

20. Venuti MG, Dhillon R, Bastiampillai T, Okungu A, Tibrewal P (2016) Cogan's syndrome and treatment-resistant psychosis. Australian and New Zealand Journal of Psychiatry 50(5): 498.

21. Dorr J, Krautwald S, Wildemann B, Jarius S, Ringelstein M, et al. (2013) Characteristics of Susac syndrome: a review of all reported cases. Nature Reviews Neurology 9(6): 307-316.

22. Hanly JG, Urowitz MB, Su L, Bae SC, Gordon C, et al. (2011) Autoantibodies as biomarkers for the prediction of neuropsychiatric events in systemic lupus erythematosus. Annals of the Rheumatic Diseases 70(10): 17261732.

23. Sciascia S, Bertolaccini ML, Roccatello D, Khamashta MA, Sanna G (2014) Autoantibodies involved in neuropsychiatric manifestations associated with systemic lupus erythematosus: a systematic review. Journal of Neurology 261(9): 1706-1714.

24. Gejman PV, Sanders AR, Kendler KS (2011) Genetics of schizophrenia: new findings and challenges. Annual Review of Genomics and Human Genetics 12: 121-144.

25. Benros ME, Pedersen MG, Rasmussen H, Eaton WW, Nordentoft M, et al. (2014) A nationwide study on the risk of autoimmune diseases in individuals with a personal or a family history of schizophrenia and related psychosis. The American Journal of Psychiatry 171(2): 218-226.

26. Michael Gusenbauer, Neal R Haddaway (2020) Which academic search systems are suitable for systematic reviews or meta-analyses? Evaluating retrieval qualities of Google Scholar, PubMed, and 26 other resources. Wiley Online Library PubMed Web 11(2): 181-217.

27. Eaton WW, Pedersen MG, Nielsen PR, Mortensen PB (2010) Autoimmune diseases, bipolar disorder, and non-affective psychosis. Bipolar Disorders 12(6): 638-646.

28. Oken RJ, Schulzer M (1999) At issue: schizophrenia and rheumatoid arthritis: the negative association revisited. Schizophrenia Bulletin 25(4): 625-638.

29. Lee SH, Byrne EM, Hultman CM, Kahler A, Vinkhuyzen AA, et al. (2015) New data and an old puzzle: the negative association between schizophrenia and rheumatoid arthritis. International Journal of Epidemiology 44(5): 1706-1721.

30. Rose NR, Bona C (1993) Defining criteria for autoimmune diseases (Witebsky's postulates revisited). Immunology Today 14(9): 426-430.

31. Saha S, Chant D, Welham J, Mcgrath JJPM (2005) A systematic review of the prevalence of schizophrenia. PLOS Medicine 2: e141.

32. Dahan S, Bragazzi NL, Yogev A, Bar-Gad M, Barak V, et al. (2018) The relationship between serum cytokine levels and degree of psychosis in patients with schizophrenia. Psychiatry Research 268: 467-472.

33. Pedersen CB, Mors O, Bertelsen A, Waltoft BL, Agerbo E, et al. (2014) A comprehensive nationwide study of the incidence rate and lifetime risk for treated mental disorders. JAMA Psychiatry 71(5): 573-581.

34. Moreno Kustner B, Martin C, Pastor L (2018) Prevalence of psychotic disorders and its association with methodological issues. A systematic review and meta-analyses. PLOS One 13(4): e0195687.

35. Pillinger T, D'ambrosio E, Mccutcheon R, Howes OD (2019) Is psychosis a multisystem disorder? A meta-review of central nervous system immune, cardiometabolic, and endocrine alterations in first-episode psychosis and perspective on potential models. Molecular Psychiatry 24(6): 776-794.
36. Momtazmanesh S, Zare-Shahabadi A, Rezaei N (2019) Cytokine alterations in schizophrenia: an updated review. Frontiers in Psychiatry 10: 892.

37. Tamminga C (2017) Schizophrenia and other psychotic disorders: Introduction and overview. In: Sadock VA, Sadock BJ, Ruiz P Md (Eds.)., Kaplan and Sadock"s Comprehensive Textbook of Psychiatry, (10 $0^{\text {th }}$ Edn.)., Wolters Kluwer Health. Lippincott Williams \& Wilkins, Philadelphia, USA, pp. 3613-3617.

38. Daros AR, Ruocco AC, Reilly JL, Harris MS, Sweeney JA (2014) Facial emotion recognition in first-episode schizophrenia and bipolar disorder with psychosis. Schizophrenia Research 153(1-3): 32-37.

39. Kohler CG, Walker JB, Martin EA, Healey KM, Moberg PJ (2010) Facial emotion perception in schizophrenia: a meta-analytic review. Schizophrenia Bulletin 36(5): 1009-1019.

40. Kohler CG, Hoffman LJ, Eastman LB, Healey K, Moberg PJ (2011) Facial emotion perception in depression and bipolar disorder: a quantitative review. Psychiatry Research 188(3): 303-309.

41. Lee J, Altshuler L, Glahn DC, Miklowitz DJ, Ochsner K, et al. (2013) Social and nonsocial cognition in bipolar disorder and schizophrenia: relative levels of impairment. The American Journal of Psychiatry 170(3): 334341.

42. Rich BA, Grimley ME, Schmajuk M, Blair KS, Blair RJ, et al. (2008) Face emotion labeling deficits in children with bipolar disorder and severe mood dysregulation. Development and Psychopathology 20(2): 529546.

43. Amador XF, Strauss DH, Yale SA, Gorman M (1991) Aware-ness of illness in schizophrenia. Schizophrenia Bulletin 17(1): 113-132.

44. Cooke MA, Peters ER, Kuipers E, Kumari V (2005) Disease, deficit or denial? Models of poor insight in psychosis. Acta Psychiatrica Scandinavica 112(1): 4-17.

45. David A (1990) Insight in psychosis. British Journal of Psychiatry 156: 798-808.

46. Amador XF, Strauss DH, Yale SA, Flaum MM, Endicott J, et al. (1993) Assessment of insight in psychosis. The American Journal of Psychiatry 150(6): 873-879.

47. Warman DM, Martin JM (2006) Cognitive insight and delusion proneness: An investigation using the Beck cognitive insight scale. Schizophrenia Research 84(2-3): 297-304.

48. Beck AT, Baruch E, Balter JM, Steer RA, Warman DM (2004) A new instrument for measuring insight: The Beck Cognitive Insight Scale. Schizophrenia Research 68(2-3): 319-329.

49. Bora E, Erkan A, Kayahan B, Veznedaroglu B (2007) Cognitive insight and acute psychosis in schizophrenia. Psychiatry and Clinical Neurosciences 61(6): 634-639.

50. Beck AT, Warman DM (2004) Cognitive insight, theory and assessment. In: Amador X, David A (Eds.)., Insight and Psychosis: Awareness of Illness in Schizophrenia and Related Disorders ( $2^{\text {nd }}$ Edn.)., Oxford University Press, Oxford, UK, p. 79-87.

51. Warman DM, Lysaker PH, Martin JM (2007) Cognitive insight and psychotic disorder; the impact of active delusions. Schizophrenia Research 90(1-3): 325-333.

52. Birchwood M, Smith V, Drury V (1994) A self-report insight scale for psychosis: Reliability, validity and sensitivity to change. Acta Psychiatrica Scandinavica 89(1): 62-67.

53. Pedrelli P, McQuaid JR, Granholm E, Patterson TL, McClure F, et al. (2004) Measuring cognitive insight in middle-aged and older patients with psychotic disorders. Schizophrenia Research 71(2-3): 297-305.

54. Ehrentheil OF (1957) Common medical disorders rarely found in psychotic patients. AMA Archives of Neurology and Psychiatry 77(2): 178-186. 
55. Pilkington TL (1956) The coincidence of rheumatoid arthritis and schizophrenia. The Journal of Nervous and Mental Disease 124(6): 604606.

56. Graff H, Handford A (1961) Celiac syndrome in the case histories of five schizophrenics. Psychiatric Quarterly 35: 306-313.

57. Hutchinson GA, Nehall JE, Simeon DT (1996) Psychiatric disorders in systemic lupus erythematosus. West Indian Medical Journal 45(2): 4850 .

58. Othman SS, Abdul Kadir K, Hassan J, Hong GK, Singh BB, et al. (1994) High prevalence of thyroid function test abnormalities in chronic schizophrenia. Australian and New Zealand Journal of Psychiatry 28(4): 620-624.

59. Reichelt KL, Landmark J (1995) Specific IgA antibody increases in schizophrenia. Biological Psychiatry 37(6): 410-413.

60. Vignesh P, Rawat A, Sharma M, Singh S (2017) Complement in autoimmune diseases. Clinica Chimica Acta 465: 123-130.

61. Zajkowska Z, Mondelli V (2014) First-episode psychosis: An inflammatory state? Neuroimmunomodulation 21(2-3): 102-108.

62. Ding M, Song X, Zhao J, Gao J, Li X, et al. (2014) Activation of Th17 cells in drug naive, first episode schizophrenia. Progress in NeuroPsychopharmacology \& Biological Psychiatry 51: 78-82.

63. Cullen AE, Holmes S, Pollak TA, Blackman G, Joyce DW, et al. (2019) Associations between non-neurological autoimmune disorders and psychosis: a meta-analysis. Biological Psychiatry 85(1): 35-48.

64. Saha S, Chant D, Welham J, McGrath J (2005) A systematic review of the prevalence of schizophrenia. PLOS Medicine 2(5): e141.

65. Eaton WW, Pedersen MG, Atladottir HO, Gregory PE, Rose NR, et al. (2010) The prevalence of 30 ICD-10 autoimmune diseases in Denmark. Immunologic Research 47(1-3): 228-231.

66. Cascella NG, Kryszak D, Bhatti B, Gregory P, Kelly DL, et al. (2011) Prevalence of celiac disease and gluten sensitivity in the United States clinical antipsychotic trials of intervention effectiveness study population. Schizophrenic Bulletin 37(1): 94-100.

67. Kalaydjian AE, Eaton W, Cascella N, Fasano A (2006) The gluten connection: The association between schizophrenia and celiac disease. Acta Psychiatrica Scandinavica 113(2): 82-90.

68. Hadjivassiliou M, Sanders DS, Grünewald RA, Woodroofe N, Boscolo S, et al. (2010) Gluten sensitivity: From gut to brain. The Lancet Neurology 9(3): 318-330.

69. Jackson JR, Eaton WW, Cascella NG, Fasano A, Kelly DL (2012) Neurologic and psychiatric manifestations of celiac disease and gluten sensitivity. Psychiatric Quarterly 83(1): 91-102.

70. Bender L (1953) Childhood schizophrenia. Psychiatric Quarterly 27: 663-681.

71. Graff H, Handford A (1961) Celiac syndrome in the case histories of five schizophrenics. Psychiatric Quarterly 35: 306-313.

72. Samaroo D, Dickerson F, Kasarda DD, Green PH, Briani C, et al. (2010) Novel immune response to gluten in individuals with schizophrenia. Schizophrenia Research 118(1-3): 248-255.
73. Sidhom O, Laadhar L, Zitouni M, Ben AN, Rafrafi R, et al. (2012) Spectrum of autoantibodies in Tunisian psychiatric inpatients. Immunological Investigations 41(5): 538-549.

74. Dickerson F, Stallings C, Origoni A, Vaughan C, Khushalani S, et al. (2010) Markers of gluten sensitivity and celiac disease in recent-onset psychosis and multi-episode schizophrenia. Biological Psychiatry 68(1): 100-104.

75. Eaton WW, Hayward C, Ram R (1992) Schizophrenia and rheumatoid arthritis: A review. Schizophrenia Research 6(3): 181-192.

76. Vinogradov S, Gottesman II, Moises HW, Nicol S (1991) Negative association between schizophrenia and rheumatoid arthritis. Schizophrenic Bulletin 17(4): 669-678.

77. Mors O, Mortensen PB, Ewald H (1999) A population-based register study of the association between schizophrenia and rheumatoid arthritis. Schizophrenia Research 40(1): 67-74.

78. Torrey EF, Yolken RH (2001) The schizophrenia-rheumatoid arthritis connection: Infectious, immune, or both? Brain, Behavior and Immunity 15(4): 401-410.

79. Benros ME, Nielsen PR, Nordentoft M, Eaton WW, Dalton SO, et al (2011) Autoimmune diseases and severe infections as risk factors for schizophrenia: A 30-year population-based register study. The American Journal of Psychiatry 168(12): 1303-1310.

80. Eaton WW, Byrne M, Ewald H, Mors O, Chen CY, et al. (2006) Association of schizophrenia and autoimmune diseases: Linkage of Danish national registers. The American Journal of Psychiatry 163(3): 521-528.

81. Margutti P, Delunardo F, Ortona E (2006) Autoantibodies associated with psychiatric disorders. Current Neurovascular Research 3(2): 149-157.

82. Eaton WW, Byrne M, Ewald H, Mors O, Chen CY, et al. (2006): Association of schizophrenia and autoimmune diseases: Linkage of Danish national registers. The American Journal of Psychiatry 163(3): 521-528.

83. Gilvarry CM, Sham PC, Jones PB, Cannon M, Wright P, et al. (1996) Family history of autoimmune diseases in psychosis. Schizophrenia Research 19(1): 33-40.

84. Yarlagadda A, Helvink B, Chou C, Clayton AH (2007) Blood brain barrier: The role of GAD antibodies in psychiatry. Psychiatry (Edgmont) 4(6): 57-59.

85. Wright P, Sham PC, Gilvarry CM, Jones PB, Cannon M, et al. (1996) Autoimmune diseases in the pedigrees of schizophrenic and control subjects. Schizophrenia Research 20(5): 261-267.

86. Feinstein A (2007) Neuropsychiatric syndromes associated with multiple sclerosis. Journal of Neurology 254: II73-II76.

87. Kosmidis MH, Giannakou M, Messinis L, Papathanasopoulos P (2010) Psychotic features associated with multiple sclerosis. International Review of Psychiatry 22(1): 55-66

88. Minagar A, Carpenter A, Alexander JS (2007) The destructive alliance: Interactions of leukocytes, cerebral endothelial cells, and the immune cascade in pathogenesis of multiple sclerosis. International Review of Psychiatry 79: 1-11.

89. Patten SB, Svenson LW, Metz LM (2005) Psychotic disorders in MS: Population-based evidence of an association. Neurology 65(7): 11231125 . 


\section{ISSN: 2574-1241}

DOI: 10.26717/BJSTR.2021.34.005619

Sumera Zaib, Imtiaz Khan. Biomed J Sci \& Tech Res

(C) This work is licensed under Creative

Submission Link: https://biomedres.us/submit-manuscript.php

$\begin{array}{ll}\text { BIOMEDICAL } & \text { Assets of Publishing with us } \\ \text { RESEARCHES } & \text { Global archiving of articles } \\ \text { - Immediate, unrestricted online access }\end{array}$

